\title{
THE EFFECT OF COUPLE-STRESSES ON THE STRESS CONCENTRATION AROUND A MOVING CRACK
}

\author{
S. ITOU \\ Department of Mechanical Engineering \\ Hachinohe Institute of Technology \\ Hachinohe 031 Japan \\ (Received March 20, 1980)
}

ABSTRACT. The problem of a uniformly propagating finite crack in an infinite medium is solved within the linearized couple-stress theory. The self-equilibrated system of pressure is applied to the crack surfaces. The problem is reduced to dual integral equations and solved by a series-expansion method. The dynamic stress-intensity factor is computed numerically. KEY WORDS AND PHRASES. Couple-Stresses, A moving crack. 1980 MATHEMATICS SUBJECT CLASSIFICATION CODES. $73 \mathrm{C}$.

1. INTRODUCTION.

The classical theory of elasticity is based on the assumption that matter is continuously distributed in any elastic body. Therefore, it is an approximation for polycrystalline metals, granular materials, porous materials, discrete mate- 
rials, steel-fiber reinforced concrete and so on, which are generally known as materials with microstructures. An attempt to drop the continuity of matter assumption is bound to make the analysis for the modified theory extremely difficult. Therefore, for materials with microstructures, some models of continua are constructed in such a way as to represent a better approximation. Among the several theories available, the linearized couple-stress theory, which was developed by Mindlin and Tiersten (1), is the simplest. In the context of this theory, a large number of analytical solutions have been published as shown in Ref. (2).

On the other hand, some research has been carried out regarding the experimental evaluations of the new material constant $l$ which was introduced in the couplestress theory. In Refs. (3) and (4), bending tests were performed on aluminium alloy plate and low-carbon steel and the upper limits of the material constant $l$ are presented. Later, Savin and his coauthors accurately determined the constant by measuring the velocity of the transverse ultrasonic wave (5). The results showed that the value for $l$ falls within the limits from $10^{-2}$ to $10^{-3} \mathrm{~mm}$ for brass, bronz, duralmin and aluminium. In this case, $l$ was approximately one order of magnitude less than the mean grain size. This means that couple-stresses do not significantly affect the stress concentrations caused by the existense of circular holes or inclusions. However, the effect of couple-stresses is serious in fracture mechanics.

According to the linear theory of elasticity, the stresses in the vicinity of the ends of the cracks are inversely proportional to the square root of $r$, the distance from the end of the crack. Stress-intensity factor $K_{i}$ is defined from the coefficient. If the plastic zone is very small relative to the size of the crack, we can assume that $K_{i}$ is proportional to the applied tensile load and is a function of the crack and specimen sizes.

In linear fracture mechanics, it is considered that when the stress-intensity 
factor $K_{\mathfrak{i}}$ reaches critical value $K_{\mathfrak{i}}^{c}$, which is called the fracture toughness value and is characteristic of the material, an unstable crack propagation occurs. This idea was established by Irwim (6) and is equivalent to Griffith's original concept (7), that a crack will begin to propagate if the elastic energy released by its growth is greater than the energy required to produce the fractured surfaces.

The stress-intensity factor calculated according to the couple-stress theory is always larger than the classical solution, and furthermore, it becomes larger as the new material constant $l$ decreases (8)(9). For this reason, crack problems in the theory of couple-stresses are very important physically (10). Nevertheless, few studies have been carried out to reveal the effects of couple-stresses on the stress-intensity factor (2), because of the severe mathematical complexity encountered in finding the solutions fit to the geometries.

In the present paper, Yoffë's model (11) is solved by the two-dimensional linearized couple-stress theory. The crack propagates only to the right, maintaining its constant length. Such an idealization will affect the magnitude of the local stress field to some extent, but does not alter the qualitative features of the stress solution (12). Application of the Fourier transformation technique reduces the problem to that of solving two dual integral equations. To solve these equations, a very simple method is used, namely, the displacement and rotation on the crack's surfaces are expanded by a series of Jacobi polynomials with the Schmidt method being employed.

Numerical calculations are carried out and compared to those given by Yoffe to clarify the influence of couple-stresses on the dynamic stress-intensity factor.

\section{FUNDAMENTAL EQUATION}

With respect to the fixed rectangular coordinate system $(\bar{x}, \bar{y}, \bar{z})$, the 
equations of motion in the plane state of strain of the linearized couple-stress theory are

$$
\begin{aligned}
& 1 / c_{L}^{2} \phi_{,{ }_{i \bar{i}}}^{*}=\phi^{*}{ }_{\bar{j} \overline{\bar{x}}}+\phi_{g \bar{z} \bar{z} \bar{z}}^{*},
\end{aligned}
$$

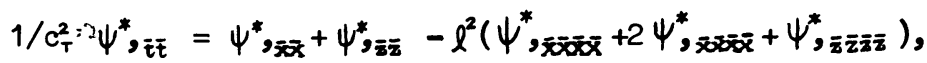

with

$$
\begin{aligned}
& u^{*}=\phi^{*}, \bar{x}-\psi_{, \bar{x}}^{*}, \\
& w^{*}=\phi^{*},_{\bar{z}}+\psi^{*}, \overline{\bar{x}},
\end{aligned}
$$

where $c_{L}=\{(\lambda+2 \mu) / \rho\}^{\frac{1}{2}}, c_{T}=(\mu / \rho)^{\frac{1}{2}} \quad$ are the dilatational and shear wave velocities, respectively. The Lamē constants are represented by $\lambda$ and $\mu, \rho$ is the density of the material, $E$ is time, $l$ is the new material's constant, $u^{*}$ and $\mathrm{w}^{*}$ are defined as the $\overline{\mathrm{x}}$ and $\overline{\mathrm{z}}$ components of the displacement, respectively, and the indices following a comma indicate the partial differentiation with respect to the variable, e.g. $\phi^{*}{ }_{\bar{x}}=\partial \phi^{*} / \partial \bar{x}_{\bullet}$. Consider the problem of an infinite elastic solid which contains a crack with a length represented by $2 a$ along the $\overline{\mathbf{x}}$ axis as shown in Fig. 1. It is assumed that the crack is opened at one end and colsed at the other with constant speed U. For a constant velocity crack, it is convenient to introduce the Galilean transformation

$$
x=\bar{x}-U E, \quad y=\bar{y}, \quad z=\bar{z}, t=E,
$$

with $(x, y, z)$ being the translating coordinate system attached to the moving crack. In the moving coordinates, the equations of motion become independent of the time variable $t$,

$$
\begin{aligned}
& \phi_{9 x x}+\phi_{g_{z z}}=M_{L}^{2} \phi_{, x x}, \\
& \psi_{\text {sxox }}+\psi_{\text {szz }}-l^{2}\left(\psi_{\text {sxoox }}+2 \psi_{\text {sxoszz }}+\psi_{\text {szzzz }}\right)=M_{T}^{2} \psi_{\text {sxx }} \text {, }
\end{aligned}
$$


where $M_{T}=U / c_{T}, M_{L}=U / c_{L}=[(1-2 \nu) /\{2(1-\nu)\}]^{\frac{1}{2}} M_{T}$ represent the Mach numbers of the moving crack with respect to longitudinal and transversal elastic waves, respectively, and $\mathcal{V}$ is the Poisson ratio.

The displacements, rotation, force-stresses and couple-stresses are expressed by the following :

$$
\begin{aligned}
& u=\phi_{\text {vx }}-\psi_{\text {ez }}, \\
& w=\phi, y+\psi g_{x} \text {, } \\
& \omega_{y}=\frac{1}{2}\left(\psi_{\text {,xx }}+\psi_{\text {gzz }_{z}}\right) \text {, } \\
& \tau_{\mathrm{zz}} / \mu=\left(\mathrm{M}_{T}^{2}-2\right) \phi_{\mathrm{gxx}}+2 \psi_{\mathrm{gxz}} \text {, } \\
& \tau_{\text {xx }} / \mu=\left(2+M_{T}^{2}-2 M_{L}^{2}\right) \phi_{\text {sxx }}-2 \psi_{\text {oxz }} \text {, } \\
& \tau_{\text {zx }} / \mu=2 \phi_{\text {sxz }}-\left(M_{T}^{2}-2\right) \psi_{\text {gxx }} \text {, } \\
& \tau_{x x} / \mu=2 \phi_{\text {gxz }}+M_{T}^{2} \psi_{\text {sxx }}-2 \psi_{\text {gzz }} \text {, } \\
& \sigma_{z} / \mu=2 l^{2}\left(\psi_{\text {gxxz }}+\psi_{\text {,zzz }}\right) \text {, } \\
& \sigma_{x} / \mu=2 l^{2}\left(\psi_{\text {gxxx }}+\psi_{\text {sxzz }}\right) \text {. }
\end{aligned}
$$

The boundary conditions for the problem to be studied are

$$
\begin{array}{rlrl}
\tau_{z z}^{0} / \mu & =-p(x), & \text { for } z=0, & |x|<a, \\
\sigma_{z}^{0} / \mu=0, & \text { for } z=0, & |x|<a, \\
w^{0}=0, & \text { for } z=0, & |x|>a, \\
\omega_{y}^{0}=0, & \text { for } z=0, & |x|>a, \\
\tau_{z x}^{0}=0, & \text { for } z=0, & |x|<\infty .
\end{array}
$$




\section{ANALYSIS}

To find the solutions for the wave equation (2.4), we use the Fourier transforms

$$
\begin{aligned}
& f(\xi)=\int_{-\infty}^{\infty} f(x) \exp (i \xi x) d x \\
& f(x)=\frac{1}{2 \pi} \int_{-\infty}^{\infty} \bar{f}(\xi) \exp (-i \xi x) d \xi
\end{aligned}
$$

Using this theory, we can reduce equation (2.4) in $x$ and $z$ to the following ordinary equation in $\mathrm{z}$

$$
\begin{aligned}
& \bar{\phi}_{\mathrm{gz}_{\mathrm{zz}}-\left(1-\mathrm{M}_{L}^{2}\right) \xi^{2} \bar{\phi}=0,} \\
& l^{2} \psi,{ }_{\mathrm{zzzz}}-\left(2 l^{2} \xi^{2}+1\right) \bar{\psi}{ }_{\mathrm{zzz}}+\left\{l^{2} \xi^{4}+\xi^{2}\left(1-M_{T}^{2}\right)\right\} \bar{\psi}=0 .
\end{aligned}
$$

Due to the symmetry conditions in equation (2.9), it is possible to consider only the problem for the half-space, $z \geqq 0$. The solutions to equation (3.2) appropriate to $\mathrm{z} \geqq 0$ will take the following forms,

$$
\begin{aligned}
& \bar{\phi}=\mathrm{A}(\xi) \exp (-\alpha z), \\
& \bar{\psi}=\mathrm{B}(\xi) \exp \left(-\beta_{1} z\right)+C(\xi) \exp \left(-\beta_{2} z\right),
\end{aligned}
$$

with

$$
\begin{aligned}
& \alpha=\left\{\left(1-M_{L}^{2}\right) \xi^{2}\right\}^{\frac{1}{2}}, \\
& \beta_{1}=\left\{2 l^{2} \xi^{2}+1+\sqrt{1+4 l^{2} \xi^{2} M_{T}^{2}}\right\}^{\frac{1}{2}} /(\sqrt{2} l), \\
& \beta_{2}=\left\{2 l^{2} \xi^{2}+1-\sqrt{1+4 l^{2} \xi^{2} M_{T}^{2}}\right\}^{\frac{1}{2}} /(\sqrt{2} \ell),
\end{aligned}
$$

where $A(\xi), B(\xi), C(\xi)$ are independent of $\mathrm{z}$.

Then, it can be shown that the mixed boundary value equation (2.9) yields the following four integral equations 


$$
\begin{aligned}
& \frac{1}{2 \pi} \int_{-\infty}^{\infty}\left\{\bar{w}^{0} k_{1}(\xi)+i \bar{\omega}_{y}^{0} k_{2}(\xi)\right\} \exp (-i \xi x) d \xi=-p(x), \\
& \frac{1}{2 \pi} \int_{-\infty}^{\infty}\left\{i \bar{\omega}^{0} k_{3}(\xi)+\bar{\omega}_{y}^{0} k_{4}(\xi)\right\} \exp (-i \xi x) d \xi=0, \quad \text { for }|x|<a,(3.5 .1) \\
& \frac{1}{2 \pi} \int_{-\infty}^{\infty} \bar{w}^{0} \exp (-i \xi x) d \xi=\frac{1}{2 \pi} \int_{-\infty}^{\infty} \bar{\omega}_{y}^{0} \exp (-i \xi x) d \xi=0, \quad \text { for }|x|>a,(3.5 .2)
\end{aligned}
$$

with

$$
\begin{aligned}
& \mathrm{k}_{1}(\xi)=\left\{l\left(M_{T}^{2}-2\right)^{2} \xi^{2}\left(\beta_{1}+\beta_{2}\right)-4 \alpha\left(l \xi^{2}+\xi \sqrt{1-M_{T}^{2}-l^{2} \xi^{2}}\right)\right\} /\left\{l M_{T}^{2} \alpha\left(\beta_{1}+\beta_{2}\right)\right\}, \\
& k_{2}(\xi)=4 \xi /\left(\beta_{1}+\beta_{2}\right), \\
& k_{3}(\xi)=-2 \xi /\left\{l^{2}\left(\beta_{1}+\beta_{2}\right)\right\}, \\
& k_{4}(\xi)=-2\left(l^{2} \xi^{2}+1+\xi l \sqrt{1-M_{T}^{2}+l^{2} \xi^{2}}\right) /\left\{l^{2}\left(\beta_{1}+\beta_{2}\right)\right\},
\end{aligned}
$$

where $\overline{\mathbf{w}}^{0}, \bar{\omega}_{\mathrm{y}}^{0}$ are the transformed displacement and rotation on $\mathrm{z}=0$, respectively. If we assume that $p(x)$ is an even function of $x$, the solutions can be represented by the series

$$
\begin{array}{ll}
w^{0}=\sum_{n=1}^{\infty} a_{n} P_{2 n-2}^{(1 / 2,1 / 2)}(x / a)\left(1-x^{2} / a^{2}\right)^{1 / 2}, & \\
w_{y}^{0}=\sum_{n=1}^{\infty} b_{n} P_{2 n-1}^{(1 / 2,1 / 2)}(x / a)\left(1-x^{2} / a^{2}\right)^{1 / 2}, & \text { for }|x|<a, \\
w^{0}=w_{y}^{0}=0, & \text { for }|x|>a,
\end{array}
$$

where $a_{n}, b_{n}$ are the coefficients to be determined and $P_{n}^{\left(V_{2}, V_{2}\right)}(x)$ is a Jacobi polynomial. The Fourier transformations of equation (3.7) are (13)

$$
\begin{aligned}
& \overline{\mathrm{w}}^{0}=2 \sqrt{\pi} \sum_{n=1}^{\infty} a_{n}(-1)^{n-1} \frac{\Gamma\left(2 n-\frac{1}{2}\right)}{(2 n-2) ! \xi} J_{2 n-1}(\xi a), \\
& \bar{\omega}_{y}^{0}=2 \sqrt{\pi} i \sum_{n=1}^{\infty} b_{n}(-1)^{n+1} \frac{\Gamma\left(2 n+\frac{1}{2}\right)}{(2 n-1) ! \xi} J_{2 n} \quad(\xi a),
\end{aligned}
$$


where $\Gamma(), J_{n}()$ are the Gamma and Bessel function, respectively. Equation (3.7) already satisfies equation (3.5.2). Substituting equation (3.8) into equation $(3.5 .1)$, we obtain for $|x|<a$,

$$
\begin{aligned}
& \sum_{n=1}^{\infty} b_{n} E_{n}(x)+\sum_{n=1}^{\infty} a_{n} F_{n}(x)=-\int_{0}^{x} p(x) d x, \\
& \sum_{n=1}^{\infty} b_{n} G_{n}(x)+\sum_{n=1}^{\infty} a_{n} H_{n}(x)=0,
\end{aligned}
$$

with

$$
\begin{aligned}
E_{n}(x) & =\frac{2(-1)^{n} \Gamma\left(2 n+\frac{1}{2}\right)}{\sqrt{\pi}(2 n-1) !}\left[\int_{0}^{\infty}\left\{k_{2}(\xi)-2\right\} / \xi^{2} J_{2 n}(\xi a) \sin (\xi x) d \xi+2\left(1-x^{2} / a^{2}\right)^{\frac{1}{2}}\right. \\
& \left.x \sin \left\{2 n \sin ^{-1}(x / a)\right\} /\left(4 n^{2}-1\right)-(x / a) \cos \left\{2 n \sin ^{-1}(x / a)\right\} /\left\{n\left(4 n^{2}-1\right)\right\}\right], \\
F_{n}(x)= & \frac{2(-1)^{n-1} \Gamma\left(2 n-\frac{1}{2}\right)}{\sqrt{\pi}(2 n-2) !}\left[\int_{0}^{\infty}\left\{k_{1}(\xi) / \xi-k_{1}^{L}\right\} / \xi J_{2 n-1}(\xi a) \sin (\xi x) d \xi\right. \\
G_{n}(x)= & \frac{(-1)^{n+1} \Gamma\left(2 n+\frac{1}{2}\right)}{(2 n-1) !}\left[\iint_{0}^{\infty}\left\{k_{4}(\xi)+2 \xi+(2 n-1) \sin \left\{(2 n-1) \sin ^{-1}(x / a)\right\}\right],\right. \\
& \left.x \sin (\xi x) d \xi-2 \sin \left\{2 n \sin _{T}^{-1}(x / a)\right\}-\left(4-M_{T}^{2}\right) /\left(4 l^{2}\right)\left[\left(1-x^{2}\right)\right\} / x^{2} / a^{2}\right)^{\frac{1}{2}} \sin \{2 n \\
& \left.\left.\times \sin (\xi(x / a)\} /\left(4 n^{2}-1\right)-x / a \cos \left\{2 n \sin ^{-1}(x / a)\right\} /\left\{2 n\left(4 n^{n}-1\right)\right\}\right]\right], \\
H_{n}(x)= & \frac{(-1)^{n-1} \Gamma\left(2 n-\frac{1}{2}\right)}{(2 n-2) !}\left[\int_{0}^{\infty}\left\{k_{3}(\xi)+1 / l^{2}\right\} / \xi J_{2 n-1}(\xi a) \sin (\xi x) d \xi\right. \\
& \left.-1 / l^{2} \sin \left\{(2 n-1) \sin ^{-1}(x / a)\right\} /(2 n-1)\right],
\end{aligned}
$$

and

$$
\mathrm{k}_{\mathrm{i}}^{L}=\left\{\left(\mathrm{M}_{\mathrm{T}}^{2}-2\right)^{2}-4 \sqrt{1-\mathrm{M}_{L}^{2}}\right\} /\left\{\mathrm{M}_{T}^{2} \sqrt{1-\mathrm{M}_{L}^{2}}\right\}
$$

where the first equation in (3.9) has been integrated with respect to $\mathrm{x}$. The functions

$$
f_{1}(\xi)=\left\{k_{2}(\xi)-2\right\} J_{2 n}(\xi a) / \xi^{2},
$$




$$
\begin{aligned}
& f_{2}(\xi)=\left\{k_{1}(\xi) / \xi-k_{1}^{2}\right\} J_{2 n-1}(\xi a) / \xi, \\
& f_{3}(\xi)=\left\{k_{4}(\xi)+2 \xi+\left(4-M_{T}^{2}\right) /\left(4 l^{2} \xi\right)\right\} J_{2 n}(\xi a) / \xi, \\
& f_{4}(\xi)=\left\{k_{3}(\xi)+1 / l^{2}\right\} J_{2 n-1}(\xi a) / \xi,
\end{aligned}
$$

behave as

$$
\begin{aligned}
& f_{1}(\xi) \rightarrow O\left(\xi^{-4.5}\right), \\
& f_{2}(\xi) \rightarrow O\left(\xi^{-3.5}\right), \\
& f_{3}(\xi) \rightarrow O\left(\xi^{-3.5}\right), \\
& f_{4}(\xi) \rightarrow O\left(\xi^{-3.5}\right), \quad \text { for large } \xi,
\end{aligned}
$$

so that the semi-infinite integrals in equation $(3.10)$ can easily be evaluated numerically by Filon's method. Equation (3.9) can be solved for the coefficients $a_{n}, b_{n}$ by a modified version of Schmit's method (14). Once the displacement and rotation at the boundary are found, this analysis is considered to be complete.

\section{STRESS-INTENSITY FACTOR}

The coefficients $a_{n}, b_{n}$ are known, so that the entire stress field is obtainable. In the fracture theory, however, the significant quantity to be calculated is the direct stress acting across the radius from the tip of the crack. It can be written as

$$
\begin{aligned}
& x=a+r \cos (\theta) \\
& z=r \sin (\theta)
\end{aligned}
$$

and the stresses for the small value of $r$ can be considered. The required stress $\tau_{\theta \theta}$ is given by 


$$
\tau_{\theta \theta}=\tau_{x x} \sin ^{2}(\theta)+\tau_{z z} \cos ^{2}(\theta)-\left(\tau_{x z}+\tau_{z x}\right) \sin (\theta) \cos (\theta)
$$

For the small value of $r$, it is shown

$$
\begin{aligned}
& \int_{0}^{\infty} \mathrm{J}_{2 n-1}(\xi a) \exp \left\{-\left(1-m^{2}\right)^{1 / 2} \mathrm{z} \xi\right\} \cos (\xi \mathrm{x}) \mathrm{d} \xi \\
& \longrightarrow(-1)^{n}\left\{\cos \theta+\left(1-\mathrm{m}^{2} \sin ^{2} \theta\right)^{1 / 2}\right\}^{\frac{1}{2}} /\left\{2 \sqrt{r / a}\left(1-m^{2} \sin ^{2} \theta\right)^{1 / 2}\right\}+0\left(r^{0}\right), \\
& \int_{0}^{\infty} \mathrm{J}_{2 n-1}(\xi a) \exp \left\{-\left(1-m^{2}\right)^{\frac{1}{2}} \mathrm{z} \xi\right\} \sin (\xi \mathrm{x}) \mathrm{d} \xi \\
& \longrightarrow(-1)^{n+1}\left\{-\cos \theta+\left(1-m^{2} \sin ^{2} \theta\right)^{1 / 2}\right\}^{\frac{1}{2}} /\left\{2 \sqrt{r / a}\left(1-m^{2} \sin ^{2} \theta\right)^{1 / 2}\right\}+0\left(r^{0}\right) .
\end{aligned}
$$

Using equations (4.2) and (4.3), we obtain the stress-intensity factor $K_{i}$,

$$
\begin{aligned}
\mathrm{K}_{i}= & \tau_{\theta \theta} \sqrt{2 \mathrm{r} / a} /\left.\mu\right|_{r \rightarrow 0} \\
= & -\sqrt{\frac{2}{\pi}} \sum_{n=1}^{\infty} a_{n} \frac{\Gamma\left(2 n-\frac{1}{2}\right)}{(2 n-2) !}\left[\frac{\left(M_{T}^{2}-2\right)\left(2+\mathrm{M}_{T}^{2}-2 M_{L}^{2}\right)}{M_{T}^{2}\left(1-\mathrm{M}_{L}^{2}\right)^{\frac{1}{2}}} \sqrt{\cos (\theta)+\mathrm{q}_{L}} \sin ^{2}(\theta) / \mathrm{q}_{L}\right. \\
& +\frac{\left(\mathrm{M}_{T}^{2}-2\right)^{2}}{\mathrm{M}_{T}^{2}\left(1-\mathrm{M}_{L}^{2}\right)^{\frac{1}{2}}} \sqrt{\cos (\theta)+\mathrm{q}_{L}} \cos ^{2}(\theta) / \mathrm{q}_{L}+\frac{4}{\mathrm{M}_{T}^{2}} \sqrt{1+\cos (\theta)}\left(\sin ^{2} \theta-\cos ^{2} \theta\right) \\
& \left.-\frac{4\left(\mathrm{M}_{T}^{2}-2\right)}{\mathrm{M}_{T}^{2}} \sqrt{\mathrm{q}_{L}-\cos (\theta)} \sin (\theta) \cos (\theta) / \mathrm{q}_{L}-\frac{8}{\mathrm{M}_{T}^{2}} \sqrt{1-\cos \theta} \sin (\theta) \cos (\theta)\right],
\end{aligned}
$$

with

$$
q_{L}=\sqrt{1-M_{L}^{2} \sin ^{2} \theta}
$$

\section{NUMERTCAL EXAMPLES AND RESULTS}

Numerical calculations arecarried out for $\nu=0.25$ and $p(x)=P$ (constant). The semi-infinite integrals which occur in equation (3.10) can easily be evaluated numerically, because the values for $f_{1}(\xi), f_{2}(\xi), f_{3}(\xi), f_{4}(\xi)$ decay rapidly when $\xi$ becomes large, as shown in Table 1. Adopting the first five terms of the infinite series of equation (3.9), we utilize the Schmidt procedure. In order to 
check on the accuracy, the values for the left-hand side in equation (3.9) are given in Table 2 for $\ell / a=0.1,0.5$ and $M_{T}=0.6$. From this, it is clear that the accuracy of Schmidt's method is satisfactory. In Table 3, the stress intensity factor $K_{i} / P$ for $\theta=0^{\circ}, M_{T}=0.01,0.6,0.8,0.9$ is shown, in which the values put in the circular-type brackets are those obtained from the diagram in Ref. (8) and the results given by Atkinson and Leppington (9) are also written in the squaretype brackets. The values for $M_{T}=0.01$ coincide well with those corresponding to the static solutions. In Figs. 2 and 3, the stress-intensity factor $K_{i}$ is plotted against $M_{T}$ for $\ell / a=0.1,0.5$ and $\theta=0^{\circ}, 27^{\circ}, 54^{\circ}$. In Fig.4, $K_{i}$ at $\theta=0^{\circ}$ is plotted against $l / a$. It is difficult to carry out numerical calculations in the $l / a<0.1$ range, because we cannot expect that the integrands in equation (3.10) to rapidly decay when $\xi$ becomes large for a small value of $l / a$. In Fig. 4, the broken lines are likely curves drawn suitably. Figure 5 shows stress-intensity factor $K_{i}$ versus $\theta$ for $l / a=0.2$ and $M_{T}=0.01,0.8,0.9$.

As a result of the above calculations, we are able to deduce the following information.

i) In contrast to the classical solution, the stress-intensity factor $K_{i}$ at $\theta=0^{\circ}$ is dependent on the propagating speed and becomes larger as the speed increased. ii) The maximum value of the stress-intensity factor $K_{\dot{l}}$ occurs at $\theta=0^{\circ}$ even though the crack speed is increased. Therefore, the crack branching does not occur for materials which take a nonzero value for $l$. iii) The moving velocity of the crack has less of an effect on stress-intensity factor $K_{i}$ when the value for $l$ increases. iv) Variations of the stress-intensity factor $K_{i}$ with the angle $\theta$ is much different from that of the classical solution. 
Table 1 Values of $f_{1}(\xi), f_{2}(\xi), f_{3}(\xi), f_{4}(\xi)$ for $n=1, M_{T}=0.6$ and $\ell / a=0.1,0.5$ against $\xi$ a.

\begin{tabular}{|c|c|c|c|c|c|}
\hline$l / a$ & $\xi a$ & $f_{1}(\xi)$ & $f_{2}(\xi)$ & $f_{3}(\xi)$ & $f_{4}(\xi)$ \\
\hline \multirow{3}{*}{0.1} & $\begin{array}{l}0.01 \\
0.21\end{array}$ & $\begin{array}{l}-0.24950 \times 10^{0} \\
-0.23880 \times 10^{\circ}\end{array}$ & $\begin{array}{l}0.11099 \times 10^{\prime} \\
0.10637 \times 10^{\prime}\end{array}$ & $\begin{array}{l}0.11350 \times 10^{2} \\
0.10821 \times 10^{2}\end{array}$ & $\begin{array}{l}0.49899 \times 10^{2} \\
0.47672 \times 10^{2}\end{array}$ \\
\hline & 40.01 & ${ }_{-0.30476 \times 10^{-8}}$ & $\begin{array}{c}\vdots \\
0.96084 \times 10^{-4}\end{array}$ & $\frac{\vdots}{0.12659 \times 10^{-6}}$ & $\begin{array}{c}\vdots \\
0.39442 \times 10^{-2}\end{array}$ \\
\hline & $80 . \dot{0} 1$ & $-0.67400 \times 10^{-7}$ & $-0.55^{\circ} 57 \times 10^{-5}$ & $0.27 \dot{2} 58 \times 10^{-5}$ & $-0.22575 \times 10^{-3}$ \\
\hline \multirow{3}{*}{0.5} & $\begin{array}{l}0.01 \\
0.21\end{array}$ & $\begin{array}{l}-0.24751 \times 10^{0} \\
-0.20116 \times 10^{\circ}\end{array}$ & $\begin{array}{l}0.11012 \times 10^{1} \\
0.90954 \times 10^{0}\end{array}$ & $\begin{array}{l}0.45002 \times 10^{0} \\
0.35930 \times 10^{0}\end{array}$ & $\begin{array}{l}0.19801 \times 10^{\prime} \\
0.16063 \times 10^{\prime}\end{array}$ \\
\hline & 40.01 & $-0.12456 \times 10^{-9}$ & $0.39156 \times 10^{-5}$ & $0.95798 \times 10^{-10}$ & $\frac{\vdots}{0.64399 \times 10^{-5}}$ \\
\hline & $80 . \dot{0} 1$ & $-0.27154 \times 10^{-8}$ & $-0.21915 \times 10^{-6}$ & $0.84226 \times 10^{-7}$ & $-0.36254 \times 10^{-6}$ \\
\hline
\end{tabular}

Table 2 Values of $\left\{\sum_{n=1}^{\infty} b_{n} E_{n}(x)+\sum_{n=1}^{\infty} a_{n} F_{n}(x)\right\} / P$ and $\left\{\sum_{n=1}^{\infty} b_{n} G_{n}(x)+\sum_{n=1}^{\infty} a_{n} H_{n}(x)\right\} / F$ for $M_{T}=0.6$ and $l / a=0.1,0.5$.

\begin{tabular}{l|l|c|c}
\hline$l / a$ & $x / a$ & $\left\{\sum_{n=1}^{\infty} b_{n} E_{n}(x)+\sum_{n=1}^{\infty} a_{n} F_{n}(x)\right\} / P$ & $\left\{\sum_{n=1}^{\infty} b_{n} G_{n}(x)+\sum_{n=1}^{\infty} a_{n} H_{n}(x)\right\} / P$ \\
\hline \multirow{4}{*}{0.1} & 0.001 & $-0.9996835 \times 10^{-3}$ & $0.1495043 \times 10^{-4}$ \\
& 0.1 & $-0.9997904 \times 10^{-1}$ & $0.1138374 \times 10^{-3}$ \\
& 0.5 & $-0.4999970 \times 10^{\circ}$ & $-0.5381461 \times 10^{-4}$ \\
& 0.9 & $-0.8999938 \times 10^{\circ}$ & $0.3729001 \times 10^{-4}$ \\
& 0.999 & $-0.9989995 \times 10^{0}$ & $-0.4340380 \times 10^{-3}$ \\
\hline \multirow{4}{*}{0.5} & 0.001 & $-0.9999725 \times 10^{-3}$ & $-0.3884164 \times 10^{-8}$ \\
& 0.1 & $-0.9999883 \times 10^{-1}$ & $-0.1314380 \times 10^{-5}$ \\
& 0.5 & $-0.4999998 \times 10^{\circ}$ & $-0.4316153 \times 10^{-6}$ \\
& 0.9 & $-0.8999987 \times 10^{0}$ & $-0.6969440 \times 10^{-6}$ \\
& 0.999 & $-0.9989992 \times 10^{\circ}$ & $-0.5124155 \times 10^{-6}$ \\
\hline
\end{tabular}


Table 3 Stress-intensity factor $K_{i} / P$ at $\theta=0^{\circ}$ for $\mathrm{M}_{\mathrm{T}}=0.01,0.6,0.8,0.9$ and $l / \mathrm{a}=0.1$, $0.2,0.5,1.0$.

\begin{tabular}{|c|c|c|c|c|}
\hline \multicolumn{2}{|c|}{$l / a=0.1$} & 0.2 & 0.5 & 1.0 \\
\hline$M_{T}=0.01$ & $\begin{array}{l}1.223 \\
(1.231) \\
{[1.22]}\end{array}$ & $\begin{array}{l}1.199 \\
(1.202) \\
{[1.20]}\end{array}$ & $\begin{array}{c}1.121 \\
(1.120)\end{array}$ & $\begin{array}{c}1.056 \\
(1.063)\end{array}$ \\
\hline 0.6 & 1.331 & 1.279 & 1.153 & 1.073 \\
\hline 0.8 & 1.553 & 1.418 & 1.193 & 1.085 \\
\hline 0.9 & 2.097 & 1.626 & 1.233 & 1.096 \\
\hline
\end{tabular}

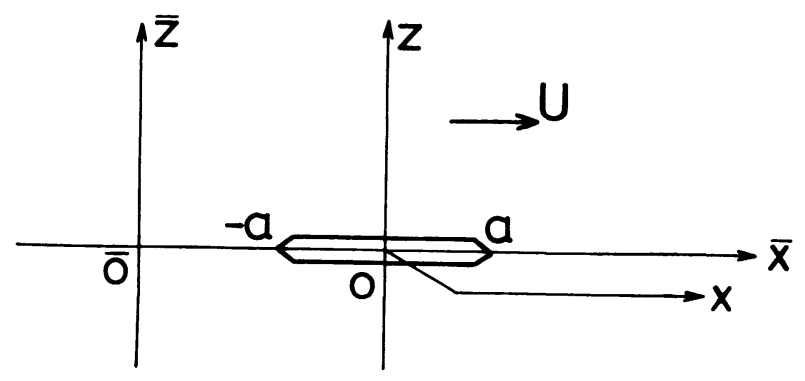

Fig. 1 Geometry and coordinate system. 


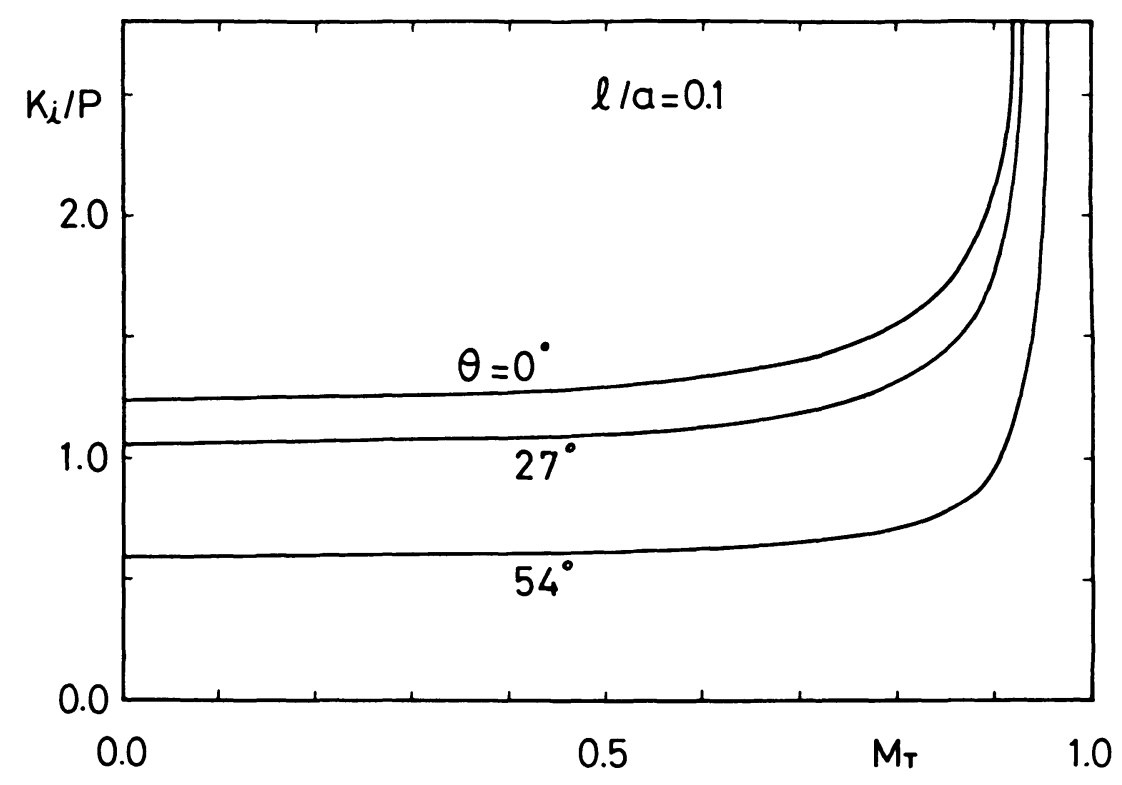

Fig. 2 Stress-intensity factor $K_{i}$ for $l / a=0.1, \theta=0^{\circ}$, $27^{\circ}, 54^{\circ}$ versus $\mathrm{M}_{\mathrm{T}}$.

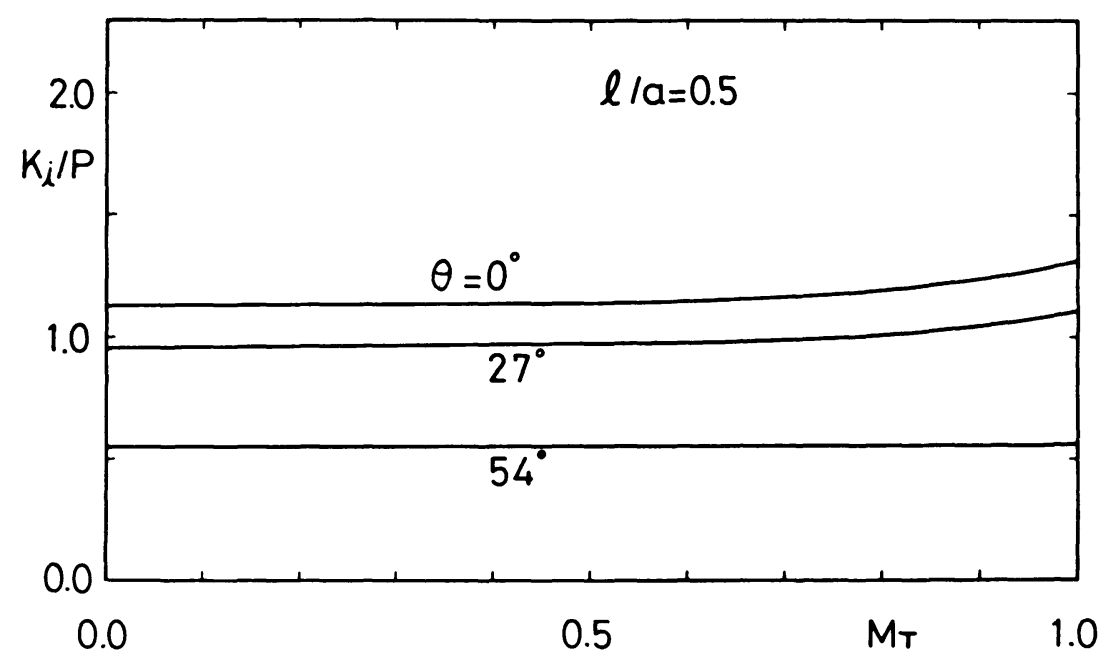

Fig. 3 Stress-intensity factor $K_{i}$ for $l / a=0.5, \theta=0^{\circ}$, $27^{\circ}, 54^{\circ}$ versus $M_{T}$. 


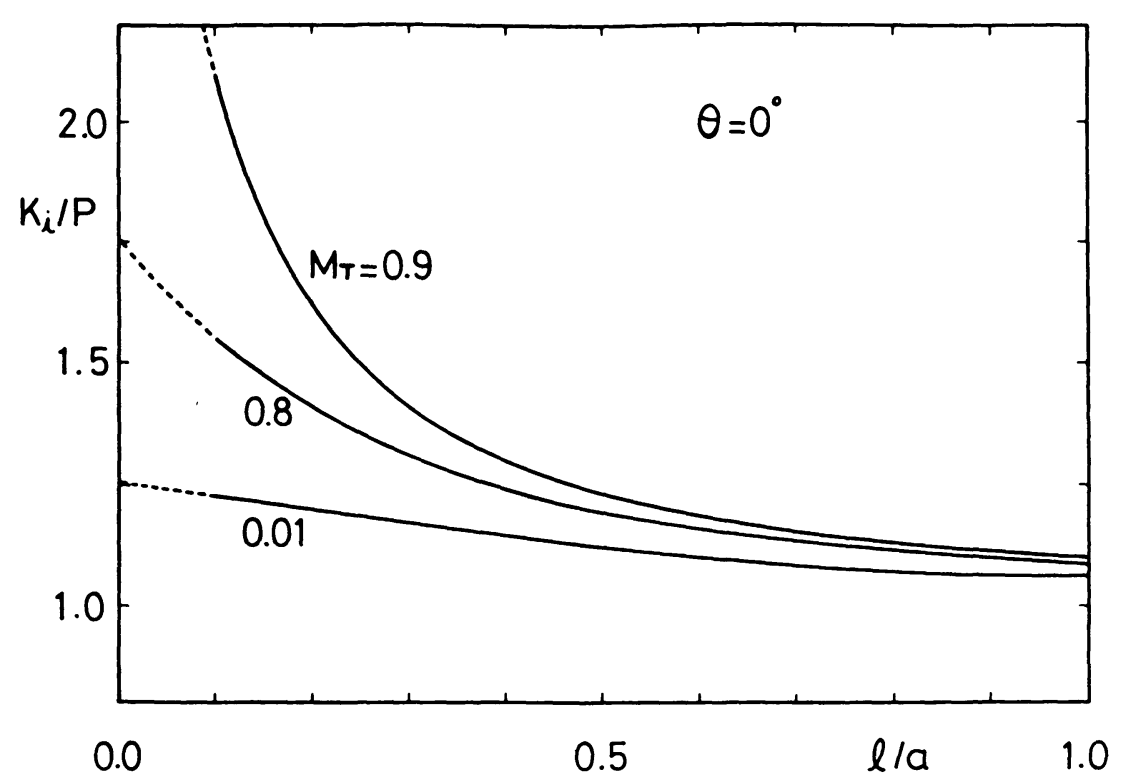

Fig. 4 Stress-intensity factor $K_{i}$ at $\theta=0^{\circ}$ for $M_{T}=0.01,0.8$, 0.9 versus $\ell / a$.

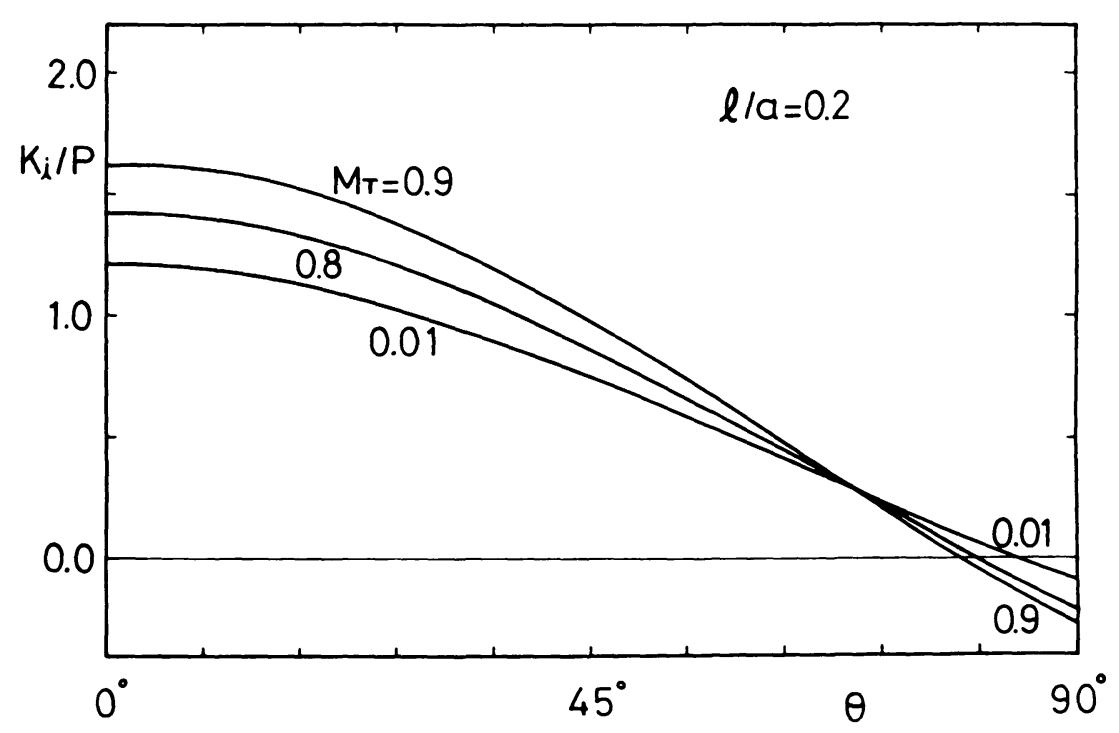

Fig. 5 Stress-intensity factor $K_{i}$ for $l / a=0.2, M_{T}=0.01$, $0.8,0.9$ versus $\theta$. 
ACKNOWLEDGEMENT: The author wishes to express his grateful thanks to Professor A. Atsumi of Tohoku University for his constant help during the preparation of the paper. This work was partially funded by the Ministry of Education.

\section{REFERENCES}

[1] Mindlin, R.D. and H.F. Tiersten, "Effects of Couple-Stresses in Linear Elasticity" Arch. Rat. Mech. Ana1. 11 (1962) 415-448.

[2] Hlavaček, M., "Vḷiv Momentovych Napěti v Problēemech Koncentrace Napěti", Strojnicky Casopis 24(1973) 373-381.

[3] Schijve, J., "Note on Couple-Stresses", J. Mech. Phys. Solids 14(1966) 113-120.

[4] E1lis, R.W. and C.W. Smith, "A Thin-plate Analysis and Experimental Evaluation of Couple-Stress Effects", Exp. Mech. (1967) 372-380.

[5] Savin, G.N., A.A. Lukasev, E.M. Lysko, S.V. Veremejenko and G.G. Agasjev, "Elastic Wave Propagation in a Cosserat Continuum with Constrained Particle Rotation", Prik1. Mekh. 6(1970) 37-41.

[6] Irwin, G.R., "Analysis of Stresses and Strains Near the End of a Crack Traversing a Plate", ASME J. App1. Mech.24(1957) 361-364.

[7] Griffith, A.A., "The Phenomena of Rupture and Flow in Solids", Phil. Trans. Roy. Soc. London, Series A, 221 (1920) 163-198.

[8] Sternberg, E. and R. Muki, "The Effect of Couple-Stresses on the Stress Concentration Around a Crack", Int. J. Solids Structures 3 (1967) 69-95.

[9] Atkinson, C. and F.G. Leppington, "The Effect of Couple-Stresses on the Tip of a Crack", Int. J. Solids Structures 13(1977) 1103-1122.

[10] Tiersten, H.F. and J.L. Bleustein, Generalized Elastic Continua, R.D. Mindlin and Applied Mechanics (Ed. by G. Herrmann), Pergamon Press, 1973, 67-103.

[11] Yoffe, E.H, The Moving Griffith Crack, Phil. Mag. 42(1951) 739-750.

[12] Sih, G.C., Some Elastodynamic Problems of Cracks, Int. J. Fracture Mech. $\underline{4}(1968) 51-68$.

[13] Erdelyi, A. (Editor), Tables of Integral Transforms, Vo1. 1, McGraw-Hill, 1954 .

[14] Yau, W.F., Axisymmetric Slipless Indentation of an Infinite Elastic Cylinder, SIAM J. App1. Math. 15(1967) 219-227. 


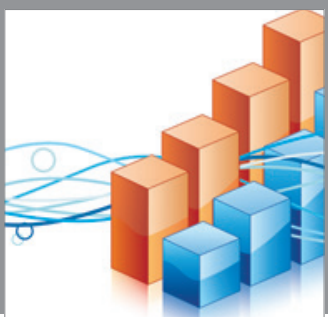

Advances in

Operations Research

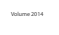

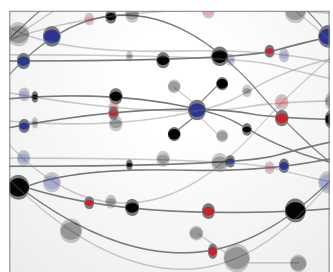

\section{The Scientific} World Journal
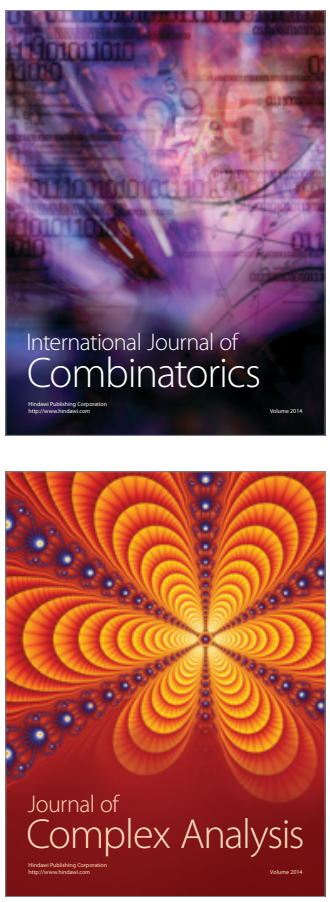

International Journal of

Mathematics and

Mathematical

Sciences
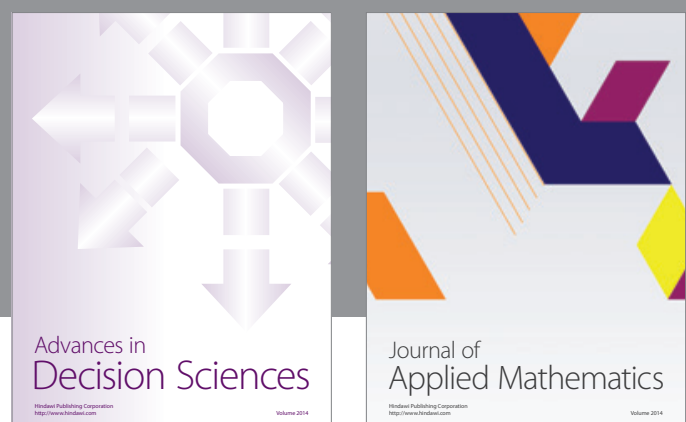

Journal of

Applied Mathematics
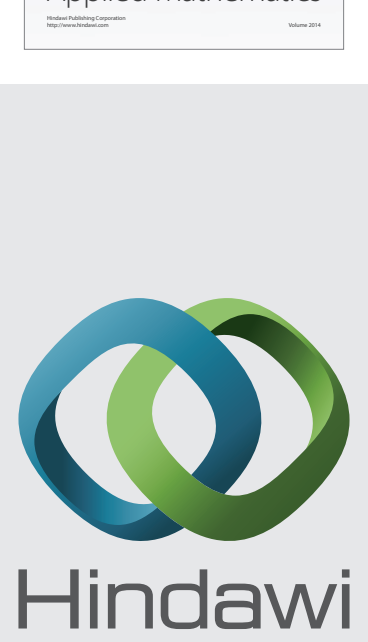

Submit your manuscripts at http://www.hindawi.com
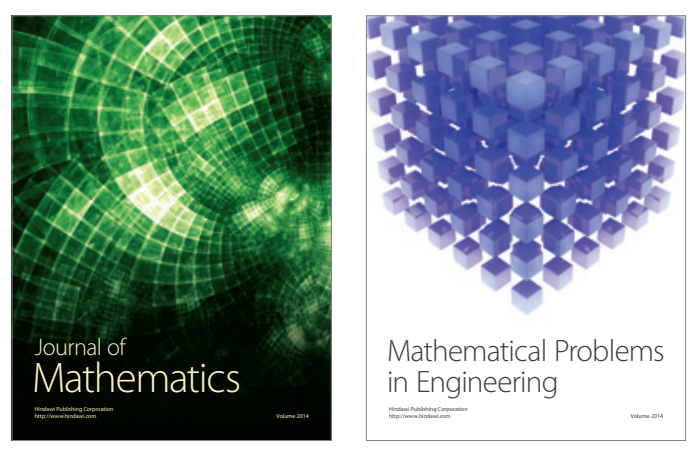

Mathematical Problems in Engineering
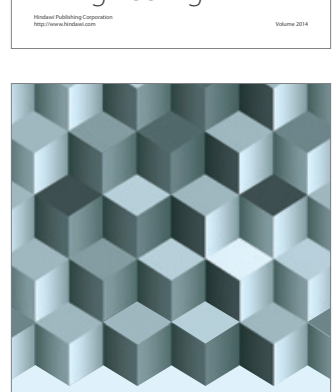

Journal of

Function Spaces
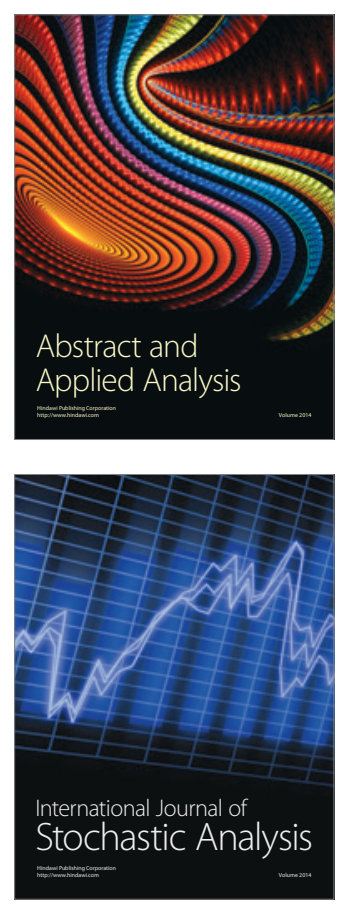

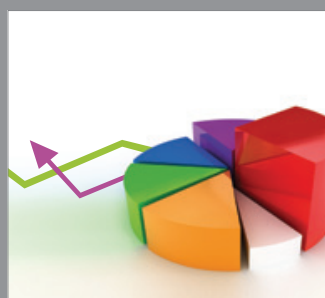

ournal of

Probability and Statistics

Promensencen
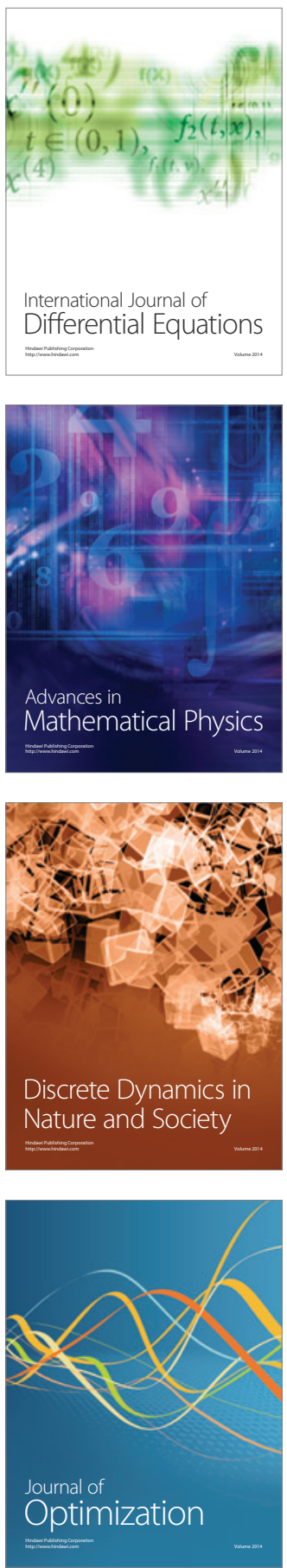